\title{
A SURVEY OF INTEGRATION BY PARTS FOR PERRON INTEGRALS
}

\author{
P. S. BULLEN \\ (Received 22 December 1983) \\ Communicated by R. O. Vyborny
}

\begin{abstract}
The history of the proof of the integration by parts formula for the classical Perron integral, and for the SCP-integral of Burkill, is discussed.
\end{abstract}

1980 Mathematics subject classification (Amer. Math. Soc.): 28 - 02.

\section{Introduction}

1. For the integral of elementary calculus the Newton, or $\mathrm{N}$-, integral-the integration by parts formula is a rewriting of the product rule of differentiation.

To say that $f$ is $\mathbf{N}$-integrable on the compact interval $[a, b], f \in \mathbf{N}(a, b)$, or just $f \in \mathbf{N}$, means $f$ is a finite derivative; that is, $f:[a, b] \rightarrow \mathbb{R}$, and there exists an $F$ such that $F^{\prime}=f$. Then $F$ is called an N-primitive of $f, F=\mathrm{N} f f$.

If $f, g \in \mathbf{N}$, with primitives $F, G$ respectively, then $(F G)^{\prime}=f G+F g$, or equivalently

$$
\mathrm{N} \int_{a}^{b} f G=F(b) G(b)-F(a) G(a)-\mathrm{N} \int_{a}^{b} F g
$$

This paper is based on talks given in May 1982 to the 5th Real Analysis Symposium, University of Missouri, Kansas City; it was written in part while the author was a visitor at the National University of Singapore, and at the University of Melbourne, 1982-83. The author would like to express his appreciation of the help and valuable comments of Professor R. O. Vyborny.

C 1986 Australian Mathematical Society $0263-6115 / 86 \$ A 2.00+0.00$ 
The same formula holds for other classical integrals, in particular the Lebesgue, or $\mathrm{L}_{-}$, integral. In that case we write (1) by dropping the prefixes $\mathrm{N}$-, (integrals without prefixes will always denote L-integrals), and a possible proof is to note that then the right-hand side of (1) is absolutely continuous, as a function of $b$, and has a derivative equal to $f G$ almost everywhere (a.e.).

The $\mathrm{L}-$ and $\mathrm{N}$-integrals arise from two very different concepts of integration. While the classes of $\mathbf{L}-$, and $\mathbf{N}$ - integrable functions, $\mathbf{L}$ and $\mathbf{N}$ say, have a large overlap, they are distinct; that is, neither $\mathbf{L}-\mathbf{N}$ nor $\mathbf{N}-\mathbf{L}$ is empty. Between 1912 and 1915 three integrals were defined that were generalizations of both the $\mathrm{L}$ - and $\mathrm{N}$-integrals. They are the restricted total, or $\mathrm{T}^{*}$-integral of Denjoy, 1912, [9], [11]; the descriptive $D^{*}$-integral of Luzin, 1912, [23]; and the Perron, or P-, integral, 1914-15, Perron [28], Bauer [2]. All three integrals turned out to be equivalent. While this is almost immediate for the first two, their equivalence to the P-integral is due to Hake [14], Looman [22], and Aleksandrov [1], 1921-25; this result is known as the Hake-Looman-Aleksandrov Theorem, or just HLA.

For the $\mathrm{T}^{*}$ - and $\mathrm{D}^{*}$-integrals the integration by parts formula is almost an immediate consequence of their definitions. The $T^{*}$-integral is defined inductively from the L-integral; starting with the formula for the L-integral, it is easily checked that the formula goes along with the induction, and the result for the $T^{*}$-integral follows. The $D^{*}$-integral is defined by generalizing the absolute continuity definition of the L-integral, and the proof suggested above for the L-integral easily generalizes to give a $\mathrm{D}^{*}$-integral integration by parts formula; the generalization of absolute continuity is usually written $\mathrm{ACG}^{*}$; see Saks [31]. (A further generalization, written ACG, defines an even more general integral, the D-integral; see also Saks [31]. This integral is mentioned in Paragraph 14 below, but nothing beyond its existence and generality is needed for this article.)

Since $\mathbf{P}=\mathbf{T}^{*}=\mathbf{D}^{*}$, either of the above results implies an integration by parts formula for the P-integral, but only by an appeal to a deep result, HLA. It is a strange fact that although the definition of the P-integral (see Paragraph 4 below) is much simpler than either of the other two definitions, no direct proof of a Perron-integral integration by parts formula became generally known until the 1967 paper of Gordon and Lasher [13]. This long delay was not due to lack of interest. At the International Congress in Oslo, 1935, McShane presented a modification of the P-integral, the MP-integral say. His purpose was that for this Perron integral a direct proof of an integration by parts formula could be given (McShane [25]). Slightly earlier, J. C. Burkill had defined a family of Perron integrals, the $\mathrm{C}_{n} \mathrm{P}$-integrals, $n \geqslant 0$, where the $\mathrm{C}_{0} \mathrm{P}$-integral is just the P-integral. For $n \geqslant 1$, Burkill gave an integration by parts formula for the $\mathrm{C}_{n} \mathrm{P}$-integral. This was the first direct proof for a Perron integral and was an important reason for the successful use made of these integrals (Burkill [6]). 
In Section 1 the problem of this long delay is discussed and it is shown that a simpler modification than the MP-integral could have been used. Also the attention of a wider audience is drawn to a simple direct proof of an integration by parts formula for the P-integral given in 1951 by Mařik [24].

Besides the original papers mentioned above, good discussions of the various integrals can be found in the following books: Hobson [16], Lebesgue [21], McShane [26], Natanson [27], Pesin [29], Saks [32]. In addition, a survey with an extensive bibliography can be found in Bullen [4].

2. The other Perron integral for which integration by parts has an interesting history arises in a very different way. (The various facts about trigonometric series that follow can be found in Zygmund [38], and an interesting discussion of this topic can be found in the first volume of Denjoy [10], and in James [19].)

Let us suppose that for all $\theta$,

$$
f(\theta)=\frac{1}{2} a_{0}+\sum_{n \geqslant 1}\left(a_{n} \cos n \theta+b_{n} \sin n \theta\right) .
$$

Then this representation of $f$ is unique. This implies that the coefficients $a_{n}, b_{n}, n \geqslant 0$, should be obtainable from $f$; in fact if, in (2) the convergence is uniform, or $f \in \mathbf{L}(-\pi, \pi)$, it is known that for any $a \in \mathbb{R}, n \geqslant 0$,

$$
a_{n}=\frac{1}{\pi} \int_{a}^{a+2 \pi} f(\theta) \cos n \theta d \theta, \quad b_{n}=\frac{1}{\pi} \int_{a}^{a+2 \pi} f(\theta) \sin n \theta d \theta .
$$

However, an $f$ given by (2) need not even be P-integrable, and in 1951 Burkill defined a Perron integral, the SCP-integral (see Paragraph 13 below) that was general enough to ensure that for $f$ given by (2), $f \in \mathbf{S C P}$. It is then fairly immediate that (3) holds for $n=0$; that is,

$$
a_{0}=\frac{1}{\pi} \mathrm{SCP} \int_{a}^{a+2 \pi} f \text {. }
$$

However, for $n \geqslant 1$, it is not at all obvious that $f \in \mathbf{S C P}$ implies $f(\theta) \cos n \theta$, $f(\theta) \sin n \theta \in$ SCP. It is this difficulty that the integration by parts formula for the SCP-integral solves, and in addition gives (3).

A natural primitive of an $f$ given by (2) is the once integrated series,

$$
F(\theta)=\frac{1}{2} a_{0} \theta+c+\sum_{n \geqslant 1} \frac{a_{n} \sin n \theta-b_{n} \cos n \theta}{n} .
$$

It is known that, given (2), this series converges a.e. to an $L$-integrable function (even to an $L_{2}$-function). So, if for simplicity we assume that $a_{0}=c=0$, then by (3) we have

$$
\frac{a_{n}}{n}=\frac{1}{\pi} \int_{a}^{a+2 \pi} F(\theta) \sin n \theta d \theta, \quad-\frac{b_{n}}{n}=\frac{1}{\pi} \int_{a}^{a+2 \pi} F(\theta) \cos n \theta d \theta .
$$


If we then assume an integration by parts formula for the SCP-integral, we could proceed to obtain (3) from (6):

$$
\mathrm{SCP} \int_{a}^{a+2 \pi} f(\theta) \cos n \theta d \theta=n \int_{a}^{a+2 \pi} F(\theta) \sin n \theta d \theta=a_{n} \pi
$$

The existence of the integral on the left-hand side of (7) ensured by that on the right-hand side, a similar argument gives the other formula (Burkill [7]).

The limits of integration in (3) are taken as shown, rather than the usual 0 and $2 \pi$, since in this application, in (4) and (7), $a$ must be a point of convergence of (5).

In Section 2 the problems encountered in proving an integration by parts formula for the SCP-integral are discussed. This section is much more technical than Section 1.

3. The use of the integration by parts formula in Paragraph 2, in particular (7), suggests that the symmetric formula (1) should, in a more general situation, be replaced by a formula in which the integral on the right-hand side exists in a stronger sense that the integral on the left-hand side:

$$
\mathrm{I} \int_{a}^{b} f G=F(b) G(b)-F(a) G(a)-\mathrm{J} \int_{a}^{b} F g
$$

where $\mathbf{J} \subset \mathbf{I}$. The formula (8) then answers the question "If $f \in \mathbf{I}$, for what $G$ is it true that $f_{G} \in \mathbf{I}$ ", the answer being those $G$ for which $F g \in \mathbf{J}$. The answer, in effect, reduces the problem for the I-integral to a similar one for the simpler J-integral.

Since for $\mathbf{I}=\mathbf{C}_{n} \mathbf{P}, n>1$, there was a natural $\mathbf{J}$ of the same type, $\mathbf{J}=\mathbf{C}_{n-1} \mathbf{P}$, Burkill was able to prove integration by parts for the $\mathbf{C}_{n} \mathbf{P}$-integral. The difficulty for $n=0$, the classical $P$-integral, was that the natural J-integral is the L-integral, an integral defined in a completely different manner.

Since it is to be expected that conditions under which (8) is proved would imply the existence of the right-hand side, in particular they would imply $F g \in \mathbf{J}$, and so $F g \in \mathbf{I}$. This leads to the simple observation that to prove (8), under those circumstances, it is sufficient to prove that $(f G+F g) \in \mathbf{I}$, and

$$
\mathrm{I} \int_{a}^{b}(f G+F g)=F(b) G(b)-F(a) G(a) .
$$

This is the form in which (1) is usually proved.

It might be mentioned that the P-integral can be defined using a modification of the Riemann integral definition. If this approach is used, the problems with the 
integration by parts formula that are being discussed do not arise; see Henstock [15] andd Pfeffer [30].

\section{The classical Perron integral}

4. The P-integral. Perron's method of major and minor functions has a wide use in the theory of linear differential equations, and in axiomatic potential theory. Its simplest, and original, application is to solve the following initial value problem: given $f:[a, b] \rightarrow \mathbb{R}$, find the unique $F$ such that, for $a \leqslant x \leqslant b$,

$$
F^{\prime}(x)=f(x) \text { and } F(a)=0 .
$$

Definition 1. If $f:[a, b] \rightarrow \mathbb{R}$, then $f \in \mathbf{P}(a, b)$ if

(I) there exist $m, M:[a, b] \rightarrow \mathbb{R}$ such that

(a) $m, M$ are continuous;

(b) $M(a)=m(a)=0$;

(c) $\mathbf{I D} M \geqslant f \geqslant \mathbf{u D} m$ a.e.;

(d) $\mathbf{I D} M>-\infty, \mathbf{u D} m<\infty$ except on a countable set.

(Here, ID denotes lower differentiation, that is to say,

$$
\operatorname{ID} M(x)=\liminf _{y \rightarrow x} \frac{M(y)-M(x)}{(y-x)} ;
$$

uD denotes upper differentiation, defined analogously; 'except on a countable set' will be written 'nearly everywhere' or just n.e.)

(II) $\sup m(b)=\inf M(b)$, where the inf is over all $M$, and the sup over all $m$, given in (I).

Then the common value in (II) is written $\mathrm{P} \int_{a}^{b} f$.

The essential properties implied by (a) to (d) are that, for all such $M$ and $m$, the function $M-m$ is increasing, continuous, and non-negative (Bruckner [3]). From these properties, and by various elementary arguments, the usual properties expected of an integral are deduced, but not the integration by parts formula.

In particular, if $f \in \mathbf{P}(a, b)$, then $f$ is finite a.e., and $f \in \mathbf{P}(a, x), a \leqslant x \leqslant b$; thus the $P$-primitive $F(x)=\mathrm{P} \int_{a}^{x} f$ is defined, $a \leqslant x \leqslant b ; F$ is continuous and $F^{\prime}=f$ a.e. If $f=g$ a.e., then $f$ and $g$ are $P$-integrable together, and when integrable they have the same primitive.

These results show that there would be no loss in generality if, in Definition 1, $f$ had been required to be finite; the extension of the integral to a.e. finite functions could then have been made in an obvious way. 
Clearly the $P$-primitive solves the initial value problem (10); for in this case $f \in \mathbf{N}(a, b)$, and an $N$-primitive of $f$ can play the role of both $M$ and $m$, so $f \in \mathbf{P}$, and its $P$-primitive is an $N$-primitive. That $f \in \mathbf{L}$ implies $f \in \mathbf{P}$ with its $L$-primitive its $P$-primitive follows from a classical result in the theory of the L-integral (Saks [32, page 191]).

The exceptional sets in (c) and (d) can, without loss of generality, be assumed to be empty (Bauer [2], Looman [22]). However, since in any particular case it is more difficult to obtain such $M$ and $m$, it is convenient to have (c) and (d) as stated.

Properties of the P-integral that depend on HLA, such as its equivalence to the $D^{*}$ - and $T^{*}$-integrals, will be considered as deep non-elementary properties. Two such properties of interest here are:

(S) Saks proved that (a) can be weakened to requiring only the measurability of $m$ and $M$ (to give the same integral of course) (Saks [32, page 247]);

(M) Marcinkiewicz proved that (II) is unnecessary, for if one $m$ and $M$ exist, then $f \in \mathbf{P}$ (Saks [32, page 253]; see also Denjoy [10, Volume IV, part 2], Sarkhel [34] and Tolstov [37]).

$M$ is called a major function, and $m$ a minor function, of $f$, on $[a, b]$; simple properties of such functions, and of the primitive $F$ of an $f \in \mathbf{P}$, imply that $M$ majorizes $F$, and that $m$ minorizes $F$. Thus, to prove $f \in \mathbf{P}$, with primitive $F$, we must, for all $\varepsilon>0$, find a majorant $M$ of $F$, and a minorant $m$ of $F$, satisfying (a) to (d), and such that $0 \leqslant M(b)-m(b)<\varepsilon$; if we are prepared to appeal to the deep result $(M)$, then the reference to $\varepsilon$ can be omitted.

5. The basic lemma. From the discussion in Paragraph 3 it would appear that we wish to prove (8) with $\mathbf{I}=\mathbf{P}$ and $\mathbf{J}=\mathbf{L}$; given the need to ensure $F g \in \mathbf{L}, F$ being continuous as we have noted in Paragraph 4, the following is the basic result we will consider.

Lemma 2. If $f \in \mathbf{P}(a, b), F=\mathrm{P} \int f$, and if $G$ is absolutely continuous, $g=G^{\prime}$ a.e. and zero elsewhere, then $f G \in \mathbf{P}(a, b)$ and

$$
\mathrm{P} \int_{a}^{b} f G=F(b) G(b)-F(a) G(a)-\int_{a}^{b} F g .
$$

A preliminary, elementary, but useful, simplification of this result can be made. First note that Lemma 2 is valid if $G$ is a constant, and that if Lemma 2 holds for $G_{1}$ and $G_{2}$ then it holds for $\lambda G_{1}+\mu G_{2}, \lambda, \mu \in \mathbb{R}$. Secondly, note that every absolutely continuous function is the difference of two increasing (or decreasing) absolutely continuous functions. From these elementary facts it is easy to see that to prove Lemma 2 it is sufficient to prove the following result. 
LEMMA 3. If $f \in \mathbf{P}(a, b), F=\mathbf{P} \int f$, and if $G$ is absolutely continuous, increasing, with $G(a)=0, g=G^{\prime}$ a.e. and zero elsewhere, then $f G \in \mathbf{P}(a, b)$ and

$$
\mathrm{P} \int_{a}^{b} f G=F(b) G(b)-\int_{a}^{b} F g \text {. }
$$

It is worth remarking that now, in Lemma $3, G \geqslant 0$ and $g \geqslant 0$.

The difficulties in proving an integration by parts formula are of two kinds. The first arises in attempting to prove Lemma 3. The almost obvious generalization, Theorem 14 below, gives rise to another difficulty which will be discussed in Paragraph 11 below.

6. Jeffery's first suggestion. It is natural, given the simplicity of Definition 1 , to attempt to prove Lemma 3 , or (12), by rewriting it as in (9) and to follow as closely as possible the proof of (1). The product rule of differentiation leads one to expect that $M G$ would be a major function of $f G+F g$ when $M$ is a major function of $f$. Similarly, if $m$ is a minor function of $f$, then $m G$ should be a minor function of $f G+F g$. If so, given $\varepsilon>0$, choose $M, m$ so that $0 \leqslant M(b)-$ $m(b)<\varepsilon$, and the $0 \leqslant M(b) G(b)-m(b) G(b)<\varepsilon G(b)$. Hence $(f G+F g) \in \mathbf{P}$, and (12) holds.

It remains then to check that $M G$ is a major function, and $m G$ is a minor function, of $f G+F g$. First note that given two real valued functions in $[a, b], \psi$ and $\phi$ say, with $\psi(x) \geqslant 0, \phi(x) \geqslant 0$ and with at least one of them continuous at $x$, then

$$
\operatorname{ID} \psi \phi(x) \geqslant \phi(x) \operatorname{ID} \psi(x)+\psi(x) \operatorname{ID} \phi(x) .
$$

Clearly $M G$ satisfies (a) and (b); further, given the conditions of Lemma 3, (13) implies that

$$
\text { ID } M G \geqslant G \operatorname{ID} M+M g
$$

since $M$ majorizes $F$, and $g \geqslant 0$, it follows from (14) that (c) for $M$ implies (c) for $M G$; that is,

$$
\text { ID } M G \geqslant f G+F g \text { a.e. }
$$

However, $g$ can be $\infty$ on a set of measure zero, and at such points, as (14) shows, ID $M G$ can be $-\infty$; hence (d) need not hold.

A similar discussion with $m G$ shows that this simple direct approach to Lemma 3 fails. All that can be proved is Lemma 3 with the extra assumption that $\left|G^{\prime}\right|<\infty$ n.e.; this was pointed out, in 1942 , by Jeffery [20].

7. Jeffery's second suggestion. Let us now consider a direct approach to Lemma 3 by trying to show that $h=f G \in \mathbf{P}(a, b)$ with the right-hand side of (12) providing the obvious primitive:

$$
H(x)=F(x) G(x)-\int_{a}^{x} F g, \quad a \leqslant x \leqslant b .
$$


If then $M$ is a major function of $f$, a suitable candidate for a major function of $h$ is

$$
R(x)=M(x) G(x)-\int_{a}^{x} M g .
$$

A minor function $r$ is defined analogously, using $m$, a minor function of $f$. That $R, r$ satisfy (a) and (b) is obvious; further, given $\varepsilon>0$, a suitable choice of $M$ and $m$ will ensure that $0 \leqslant R(b)-r(b)<\varepsilon$. It remains then to check (c) and (d).

Let $x, x+h$ be in $[a, b]$; then, by the mean value theorem for the L-integral there is a $y$, between $x$ and $x+h$, such that

$$
\begin{aligned}
R(x+h)-R(x)= & M(x+h) G(x+h)-M(x) G(x) \\
& -M(y)\{G(x+h)-G(x)\},
\end{aligned}
$$

or, on rewriting,

$$
\begin{aligned}
\frac{R(x+h)-R(x)}{h}= & G(x+h) \frac{\{M(x+h)-M(x)\}}{h} \\
& -\frac{G(x+h)-G(x)}{h}\{M(y)-M(x)\} .
\end{aligned}
$$

If $G^{\prime}(x)$ if finite, and so finite a.e., (18) implies that $\operatorname{ID} R(x)=G(x) \operatorname{lD} M(x)$, and so, since $G \geqslant 0$, (c) for $M$ implies (c) for $R$; that is, $\operatorname{ID} R \geqslant f G$ a.e.

If, however, $G^{\prime}(x)=\infty$, then the second term on the right of (18) shows that ID $R(x)=-\infty$ is possible. As this can happen on a set of measure zero, and not just on a countable set, $R$ does not satisfy (d).

A similar discussion with $r$ shows that this approach to Lemma 3 fails for the same reason as in Paragraph 6; as there, this method will prove Lemma 3 if the assumption $\left|G^{\prime}\right|<\infty$ n.e. is added (Jeffery [20]).

8. The McShane approach. Definition 1 is clearly very well adapted to solving the initial value problem (10). In both (10) and Definition 1 ordinary differentiation can be replaced by any generalization to obtain a generalized Perron integral, an integral that solves the corresponding generalization of (10), provided that two conditions are satisfied: (i) the $F$ in the generalized (10) must be unique; and (ii) $M-m$ must be increasing.

A simple such generalization is to use one-sided derivatives; proofs that conditions (i) and (ii) are satisfied can be found in Bruckner [3]. Let us speak of right major and minor functions when right differentiation is used, and likewise left major and minor functions. The use of left major and minor functions defines a Perron integral, the $P_{-}$-integral, say; if we use right major and minor functions, we get another Perron integral, the $P_{+}$-integral, say. Ridder [31] and Ionescu-

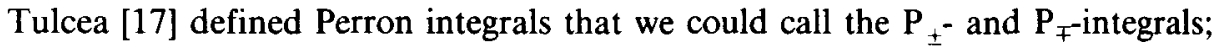


in the first, right major functions but left minor functions are used; in the second, left major functions but right minor functions are used. All four of the integrals are apparently more general than the P-integral since a (bilateral) major function is of course both a left and right major function.

The MP-integral of McShane is less general than these integrals, but still apparently more general than the P-integral. In the present notation it could be called the $\mathbf{P}_{ \pm \mp}$-integral, since the definition requires left and right major functions, and left and right minor functions. Then $f \in$ MP means that for all $\varepsilon>0$ there exist $\phi_{i}, 1 \leqslant i \leqslant 4$, left, right major and minor functions, such that $\left|\phi_{i}(b)-\phi_{j}(b)\right|<\varepsilon, 1 \leqslant i, j \leqslant 4$. (It should be noted that both for the MP-integral, and for the $\mathrm{P}_{ \pm^{-}}$, and $\mathrm{P}_{\mp}$-integral, the proof of (ii) above is more difficult. If, for instance, $M$ is a right, major function and $m$ a left minor function, appeal must be made to a result of G. C. Young; see Hobson [16, Volume 1, page 392] or Bruckner [3, page 63].)

McShane exploited the one-sided derivatives and the existence of four major, minor functions to prove Lemma 2 for the MP-integral (McShane [25], [26]). However, if we are prepared to use one-sided derivatives, the argument in Paragraph 7 gives no trouble. For let us consider (18) again and rewrite it as

$$
\begin{aligned}
\frac{R(x+h)-R(x)}{h}= & G(x+h) \frac{(M(x+h)-M(x))}{h} \\
& -(G(x+h)-G(x)) \frac{(M(y)-m(x))}{y-x} \frac{(y-x)}{h} .
\end{aligned}
$$

Now if $h<0$, then $x+h<y<x$, and $0<y-x / h<1$; if then ID_M(x) $>$ $-\infty$, (19) implies, since $G$ is increasing, that ID_ $R(x)>-\infty$. In other words, if $M$ is a (left) major function of $f$ then $R$ is a left major function of $f G$. In a similar way $r$ is a left minor function of $f G$.

In this way we obtain a very simple proof of Lemma 3, and so of Lemma 2, for the $P_{-}$-integral; and a similar argument can be given for the $P_{+}$-integral.

The $P_{+}$-integral was introduced by the Hake [14] who, as his contribution to HLA, proved that $\mathbf{P}_{+} \subset \mathbf{T}^{*}$, a result that is apparently stronger than $\mathbf{P} \subset \mathbf{T}^{*}$. Looman proved that $\mathbf{T}^{*} \subset \mathbf{P}_{+}$, and Aleksandrov that $\mathbf{T}^{*}=\mathbf{P}$. So it is a part of HLA that $\mathbf{P}_{+}=\mathbf{P}$, and so we have in fact proved Lemma 2. It is a little surprising that the above proof of Lemma 2 via the $\mathbf{P}_{+}$-integral was not noticed earlier.

Since then $\mathbf{P}_{+}=\mathbf{P}$, it follows that $\mathbf{M P}=\mathbf{P}$, and so, by an appeal to the deep HLA, McShane had given a proof of Lemma 2. (It might be remarked that the integrals $\mathbf{P}_{ \pm}, \mathbf{P}_{\mp}$ of Ridder and Ionescu-Tulcea are proper generalizations of the P-integral.) However, assuming that HLA only showed that $\mathbf{P}=\mathbf{T}^{*}$, McShane [25] showed that his integral was equivalent to the P-integral by generalizing $(M)$ (his extension of $(M)$ actually proves that $\mathbf{P}_{+}=\mathbf{P}$.) He was of the opinion that 
the equivalence $\mathbf{M P}=\mathbf{P}$, like the equivalence $\mathbf{P}_{+}=\mathbf{P}$, needs an appeal to a deep theorem (McShane [26, page 316]); however, Ridder [31] gave a very simple and elementary proof that shows McShane's proof of Lemma 2 for the MP-integral is really one for the P-integral that does not appeal to HLA. This was not noticed, and so further attempts to find a simple direct proof were made.

9. The Gordon and Lasher proof of Lemma 3. The direct proof of Lemma 3 given by Gordon and Lasher also obtains (d) by considering left and right sides separately. However, they first made a simple, but crucial observation, that they credit to Zygmund.

Since, in Lemma $3, G \geqslant 0$ and $g \geqslant 0$, and since, following remarks in Paragraph 5, we are seeking majorant of $H$ (15), the function $R$ in (16) is not a natural choice; rather we should use

$$
S(x)=M(x) G(x)-\int_{a}^{x} m g,
$$

where $M$ is a major function, and $m$ a minor function, of $f$. In a similar way we can define a candidate for a minor function $s$.

As for $R(r)$, we need only check that $S(s)$ satisfies (c) and (d). From (17), as modified for $S$, we can write

$$
\begin{aligned}
\frac{S(x+h)-S(x)}{h}= & G(x+h) \frac{(M(x+h)-M(x))}{h} \\
& -\frac{(G(x+h)-G(x))}{h}(m(y)-m(y x)) \\
& +\frac{(G(x+h)-G(x))}{h}(M(x)-m(x)) .
\end{aligned}
$$

The last term on the right-hand side of (20) is non-negative, and so if $G^{\prime}(x)$ is finite, (and so a.e.), (20) implies that

$$
\operatorname{ID} S(x) \geqslant G(x) \geqslant \operatorname{ID} M(x),
$$

and (c) follows for $S$ as it did for $R$.

As (18) was rewritten to give (19), so (20) can be rewritten as

$$
\begin{aligned}
\frac{S(x+h)-S(x)}{h}= & G(x+h) \frac{(M(x+h)-M(x))}{h} \\
& -(G(x+h)-G(x)) \frac{(m(y)-m(x))}{y-x} \frac{(y-x)}{h} \\
& +\frac{(G(x+h)-G(x))}{h}(M(y)-m(y)) .
\end{aligned}
$$

Suppose now that $h>0, \operatorname{uD} m(x)<\infty$, and $\operatorname{ID} M(x)>-\infty$ (and so n.e.). Then, as in the discussion of (19), ID $S(x)>-\infty$. 
Now rewrite (20) as

$$
\begin{aligned}
\frac{S(x+h)-S(x)}{h}= & G(x+h) \frac{(M(x+h)-M(x))}{h} \\
& -(G(x+h)-G(x))(M(y)-m(x)) \frac{(y-x)}{h} \\
& +\frac{(G(x+h)-G(x))}{h}(M(y)-m(y)) .
\end{aligned}
$$

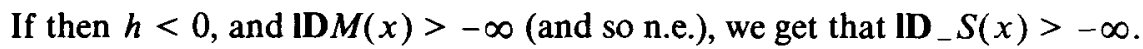

Hence ID $S>-\infty$ n.e., and $S$ is a major function of $f G$. In a similar way, $s$ is a minor function of $f G$, and the proof of Lemma 3 is complete.

10. Mařik's proof of Lemma 3. Marík's elegant proof of the integration by parts formula for the Perron integral was the first to be published and was known even earlier through mimeographed lecture notes. However, both the lecture notes and the 1951 paper were in Czech and so this proof has not received the attention it deserves.

Let us first remark that in Lemma 2, (11) can be rewritten as

$$
\mathrm{P} \int_{a}^{s} f G=F(b) G(b)-F(a) G(a)-\mathrm{RS} \int_{a}^{s} F d G,
$$

where the integral on the right-hand side of (21) is a Riemann-Stieltjes integral. It is in this form that Marík's considered the integration by parts formula for the P-integral.

It is a trivial remark that if $M$ satisfies (a), (c) and (d) of Definition 1, but not necessarily (b), then $M-M(a)$ is a major function of $f$; let us call such functions $M$ 'major' functions of $f$, with a similar definition of 'minor' functions.

Let $a<c<b$, with $M_{1}$ a 'major' function of $f$ on $[a, c]$ and $M_{2}$ a 'major' function of $f$ on $[c, b]$. Simple calculations show that

$$
M(x)= \begin{cases}M_{1}(x)-M_{1}(a), & a \leqslant x \leqslant c, \\ M_{2}(x)-M_{2}(c)+M_{1}(c)-M_{1}(a), & c \leqslant x \leqslant b,\end{cases}
$$

is a major function of $f$ on $[a, b]$, and that

$$
M(b)=\left(M_{1}(c)-M_{1}(a)\right)+\left(M_{2}(b)-M_{2}(c)\right) \text {. }
$$

More generally, if $a=c_{0}<c_{1}<\cdots<c_{n} \approx b$, and if $M_{k}$ is a 'major' function of $f$ on $\left[c_{k-1}, c_{k}\right], 1 \leqslant k \leqslant n$, then there is a major function $M$ of $f$ on $[a, b]$ such that

$$
\boldsymbol{M}(b)=\sum_{k=1}^{n}\left\{M_{k}\left(c_{k}\right)-M_{k}\left(c_{k-1}\right)\right\} .
$$

Now partition $[a, b]$ as $a=c_{0}<c_{1}<\cdots<c_{n}=b$, and let $\mu_{k}=\inf \{F(x)$; $\left.c_{k-1} \leqslant x \leqslant c_{k}\right\}$; then the lower RS-sum for the integral on the right-hand side of 
(21), for this partition, is

$$
\sum_{k=1}^{n} \mu_{k}\left\{G\left(c_{k}\right)-G\left(c_{k-1}\right)\right\}
$$

If then $M$ is a major function of $f$ on $[a, b]$, consider the expression

$$
m(b) G(b)-M(a) G(a)-\sum_{k=1}^{n} \mu_{k}\left\{G\left(c_{k}\right)-G\left(c_{k-1}\right)\right\} .
$$

Simple rewriting of (23) leads to the equivalent expression

$$
\sum_{k=1}^{n}\left[G\left(c_{k}\right)\left(M\left(c_{k}\right)-\mu_{k}\right)-G\left(c_{k-1}\right)\left(M\left(c_{k-1}\right)-\mu_{k}\right)\right],
$$

and comparing this with (22) we see that (23) is $Q(b)$ for a major function $Q$ of $f G$ on $[a, b]$, provided that, for each $k, 1 \leqslant k \leqslant n$,

$$
M_{k}=G\left(M-\mu_{k}\right)
$$

is a 'major' function of $f G$ on $\left[c_{k-1}, c_{k}\right]$. As (a) is obvious, we need only check that $M_{k}$ satisfies (c) and (d). From (13) we have

$$
\begin{aligned}
\mathbf{I D} M_{k} & \geqslant G \cdot \mathbf{I D}\left(M-\mu_{\kappa}\right)+\left(M-\mu_{k}\right) \cdot \mathbf{I D} G . \\
& =G \cdot \mathbf{I D} M+\left(M-\mu_{k}\right) \cdot \mathbf{I D} G \\
& \geqslant G \cdot \mathbf{I D} M+(M-F) \cdot \mathbf{I D} G \\
& \geqslant G \cdot \mathbf{I D} M
\end{aligned}
$$

so (c) and (d) follow for $M_{k}$, from the similar properties of $M$.

In a similar way, using a minor function of $f, m$ say, and an upper RS-sum, the expression analogous to (23) is equal to $q(b)$ for some minor function $q$ of $f G$ on $[a, b]$. Clearly $Q(b)-q(b)$ is just the difference between the upper and lower RS-sums, plus $(M(b)-m(b)) G(b)$. Since, from the hypotheses of Lemma 3, $f \in \mathbf{P}$, and since the RS-integral on the right-hand side of (21) exists, we can, given $\varepsilon>0$, find $Q, q$ such that $0 \leqslant Q(b)-q(b)<\varepsilon$. This implies that $f \in \mathbf{P}$, gives (21), and so completes Maŕík's proof of Lemma 3.

11. The general integration by parts formula. A consideration of (21) and Marík's proof suggests that the correct generalization of Lemma 2 is the following theorem.

TheOREM 4. If $f \in \mathbf{P}(a, b)$, if $F=\mathbf{P} \int f$, and if $G$ is of bounded variation, then $f G \in \mathbf{P}(a, b)$, and (21) holds.

The conditions of the theorem ensure the existence of the right-hand side of (21), which has the following interpretation:

$$
G(a)=G(a+) ; \quad G(b)=G(b-) ; \quad \operatorname{RS} \int_{a}^{b} F d G=\lim _{\substack{\alpha \rightarrow a+\\ \beta \rightarrow b-}} \operatorname{RS} \int_{\alpha}^{\beta} F d G .
$$


In the case of the $T^{*}$ - and $D^{*}$-integrals, the proofs of Theorem 4 follow the methods suggested in Paragraph 1 for the simpler Lemma 2; see for instance, Denjoy [10, Volume IVb], [11], Hobson [16] and Saks [32]. As with Lemma 2 it is sufficient to consider Theorem 4 under the extra assumptions of $G$ increasing with $G(a)=0$. If then we assume $G$ to be continuous, only minor changes in the discussions in Paragraphs 8 to 10 are needed to give proofs of Theorem 4. However, if $G$ is not assumed to be continuous, while the considerations of (b), (c) and (d) can proceed as in Paragraphs 8 to 10 Condition (a), which was trivial, now needs to be considered. The function $R$ in (16) is continuous, since (17) can be rewritten as

$$
\begin{aligned}
R(x+h)-R(x)= & (M(x+h)-M(y))(G(X+h)-G(X)) \\
& +G(x)(M(x+h)-M(x)) .
\end{aligned}
$$

Hence the discussion in Paragraph 7 will provide a proof of Theorem 4 under the added assumption of $\left|G^{\prime}\right|<\infty$ n.e. (Jeffery [18]); and McShane's proof in Paragraph 8, together with MP $=\mathbf{P}$, gives Theorem 4 for the P-integral. However, neither the function $M G$ of Paragraph 6, the function $S$ in Paragraph 9, nor the functions $M_{k}$ in Paragraph 10 are necessarily continuous; in particular, neither the Gordon and Lasher, nor the Mařik's proof of Lemma 2 extends easily to Theorem 4.

If we use (S) in Paragraph 4 above, then of course (a) would again be trivial. While this means appealing to a deep theorem, it could be reasonably argued that measurable major and minor functions are both easier and more natural in Definition 1 . Then the fact that the same integral is obtained by using continuous major and minor functions would be a deep property of the integral which is not needed to prove the integration by parts formula. This was the attitude taken in the work of Marík.

However, Theorem 4 can be proved from Lemma 2 without appealing to $(S)$, to any other deep theorem, or to the equivalence $\mathbf{M P}=\mathbf{P}$. First note that every increasing, bounded $G$, with $G(a)=0$, is the sum of two such functions, one being continuous, and the other being a saltus function. Then, arguing as we did to reduce Lemma 2 to Lemma 3, and noting that we have disposed of Theorem 4 in the case of continuous $G$, we see that it remains to consider that result for $G$ an increasing, bounded, saltus function with $G(a)=0$; further, the particular case when $G$ only has a finite number of jumps is trivial.

To complete the discussion we appeal to the following very interesting theorem due to Gordon and Lasher [13].

THEOREM 5. If $f \in \mathbf{P}(a, b)$, if $F=\mathrm{P} \int f$, if $G$ is an increasing, bounded function with $G(a)=0$, and if $k>\omega(F)=\max F-\min F$, then there exists a major function $R$ and a minor function $r$ of $f G$ such that $|R| \leqslant k G(b)$, and $|r| \leqslant k G(b)$. 
It might be remarked that Theorem 5, together with (M) in Paragraph 4, implies that, under the conditions of Theorem 4,

$$
f G \in P \text { and }\left|P \int_{a}^{b} f G\right|<S \omega(F) \cdot \operatorname{variation}(G) .
$$

Proof. It follows from Definition 1 that, for $a \leqslant x \leqslant b$,

$$
F(x)=\inf M(x)=\sup m(x),
$$

where the inf is taken over all major functions, and the sup over all minor functions.

Hence, given $k$ as in the theorem, a major function $M_{0}$ and a minor function $m_{0}$ can be chosen so that $\omega\left(M_{0}\right) \leqslant k$, and $\omega\left(m_{0}\right) \leqslant k$. Now define

$$
\begin{aligned}
& \mu(u, x)= \begin{cases}\min \left\{M_{0}(y): x \leqslant y \leqslant u\right\}, & a \leqslant x \leqslant u, \\
M_{0}(x), & u \leqslant x \leqslant b ;\end{cases} \\
& \nu(u, x)= \begin{cases}\max \left\{m_{0}(y): x \leqslant y \leqslant u\right\}, & a \leqslant x \leqslant u, \\
m_{0}(x), & u \leqslant x \leqslant b ;\end{cases}
\end{aligned}
$$

The required major and minor functions $R, r$ are then given by

$$
\begin{gathered}
R(x)=\int_{a}^{b}[\mu(u, x)-\mu(u, a)] d G(u), \\
r(x)=\int_{a}^{b}[\nu(u, x)-\nu(u, a)] d G(u) .
\end{gathered}
$$

It suffices to check that $R$ is a major function of $f G$, and that $|R| \leqslant k G(b)$, as the discussion for $r$ is similar.

The inequality is immediate, since for all $u$, we have $\omega(\mu(u, v)) \leqslant \omega\left(M_{0}\right)$; further, since $\mu$ is uniformly continuous, $R$ is continuous, and obviously $R(a)=0$. So it remains to check (c) and (d) of Definition 1. If $a \leqslant s \leqslant t \leqslant b$, then

$$
\begin{aligned}
R(t)-R(s) & =\left(\int_{a}^{s}+\int_{s}^{t}+\int_{t}^{b}\right)[\mu(u, t)-\mu(u, s)] d G(u) \\
& \geqslant\left[M_{0}(t)-M_{0}(s)\right] G(t) .
\end{aligned}
$$

Hence if $G$ is continuous at $x, a<x<b$, then $\operatorname{ID} R(x) \geqslant G(x) \operatorname{ID} M_{0}(x)$, and this suffices to show that (c) and (d) hold.

Theorem 5 has the following implication, useful for our purposes.

Corollary 6. If $G$ is such that for all $\varepsilon>0$ there exist $G_{1}, G_{2}$ such that $G=G_{1}+G_{2}$ and $(\alpha)$ Theorem 4 holds with $G_{1},(\beta) G_{2}$ satisfies the conditions of Theorem 5, and $(\gamma)\left|G_{1}\right|<\varepsilon$, then Theorem 4 holds for $G$. 
Proof. Given $\varepsilon>0$, write $G=G_{1}+G_{2}$ as above; by $(\alpha), f G_{1} \in \mathbf{P}$, so let $R_{1}\left(r_{1}\right)$ be a major (minor) function of $f G_{1}$ with $0 \leqslant R_{1}(b)-r_{1}(b)<\varepsilon$. Let $R_{1}\left(r_{2}\right)$ be the major (minor) function of $f G_{2}$ given by $(\beta)$, as in Theorem 5; put $R=R_{1}+R_{2}, r=r_{1}+r_{2}$.

Clearly $R(r)$ is a major (minor) function of $f G$, and by $(\gamma)$, and by the property of $R_{2}\left(r_{2}\right)$ from Theorem 5 , we have $0 \leqslant R(h)-r(h)<\varepsilon(1+2 k)$. Hence $f G \in \mathbf{P}(a, b)$, and since by $(\alpha), f G_{1} \in \mathbf{P}(a, b)$, it follows that $f G_{2} \in$ $\mathbf{P}(a, b)$; further, by $(\beta)$, and by Theorem $5,\left|\mathbf{P} \int_{a}^{b} f G_{2}\right|<\varepsilon k$. Hence, by (a),

$$
\begin{aligned}
& \mid \mathrm{P} \int_{a}^{b} f G-F(b) G(b)+ F(a) G(a)+\mathrm{RS} \int_{a}^{b} F d G \mid \\
&=\left|\mathrm{P} \int_{a}^{b} f G_{2}-F(b) G_{2}(b)+\mathrm{RS} \int_{a}^{b} f d G_{2}\right| \leqslant 3 \varepsilon k,
\end{aligned}
$$

and so (21) holds, and the corollary is proved.

In our case $G$ is an increasing, bounded saltus function with $G(a)=0$. If $\varepsilon>0$, we can write $G=G_{1}+G_{2}$, where $G_{1}, G_{2}$ have those same properties, with $G_{1}$ only having a finite number of jumps, and with $\left|G_{2}\right|<\varepsilon$. From the remarks above, the conditions of Corollary 6 are satisfied, and so Theorem 4 follows.

12. Concluding remarks. Theorem 4 provides the full answer to the question raised in Paragraph 3. If $f \in \mathbf{P}(a, b)$, and if $G$ is of bounded variation, then $f G \in \mathbf{P}(a, b)$. Clearly this can be extended to allow $G$ to be of essential bounded variation; that is, $G=\tilde{G}$ a.e., where $\tilde{G}$ is of bounded variation. This result cannot be generalized; Sargent [33] has shown that if $G$ is not of essential bounded variation, then there exists an $f \in \mathbf{P}$, in fact even a Cauchy-Riemann integrable $f$, such that $f G \notin \mathbf{P}$.

\section{The SCP-integral}

13. The SCP-integral. The motivation for the concepts involved in the defintion of the SCP-integral comes from the theory of trigonometric series. Since (5) converges a.e. to $F \in \mathbf{L}$, it can be integrated term-by-term to obtain

$$
\int F(\theta) d \theta=\phi(\theta)=\frac{1}{4} a_{0} \theta^{2}+c \theta+d-\sum_{n \geqslant 1} \frac{a_{n} \cos n \theta+b_{n} \sin n \theta}{n^{2}}
$$

a function that is continuous and smooth; $\phi^{\prime}(\theta)=F(\theta)$ at all points of convergence of (5) (in particular a.e.), and $D^{2} \phi=f$, where $f$ is given by (2). Here

$$
D^{2} \phi(x)=\lim _{h \rightarrow 0} \frac{\Delta^{2} \phi(x, h)}{h^{2}}, \quad \Delta^{2} \phi(x, h)=\phi(x+2 h)+\phi(x-2 h)-2 \phi(x)
$$


and $\phi$ smooth at $x$ means

$$
\lim _{h \rightarrow 0} \frac{\Delta^{2} \phi(x, h)}{h}=0 ;
$$

for details of these results see Zygmund [38]. All these properties of $\phi$ can be translated into properties of the function $F$ given by (5), which is the point of the following definition.

Definition 7. If $F \in \mathbf{P}(a, b), x \in[a, b]$, then

(a) $F$ is SC-continuous, (symmetrically Cesàro continuous), at $x$ if

$$
\lim _{h \rightarrow 0} \frac{1}{h} P \int_{0}^{h}\{F(x+u)-F(x-u)\} d u=0 .
$$

(b) $F$ is $C$-continuous at $x$ if

$$
\lim _{h \rightarrow 0} \frac{1}{h} \mathrm{P} \int_{0}^{h}(F(x+u)-F(x)) d u=0 ;
$$

(c) $F$ is SC-differentiable at $x$ if the following limit exists:

$$
\lim _{h \rightarrow 0} \frac{1}{h^{2}} \mathbf{P} \int_{0}^{h}\{F(x+u)-F(x-u)\} d u .
$$

The value of that limit is the SC-derivative of $F$ at $x$, denoted $\operatorname{SCD} F(x)$.

If $F$ is given by (5) it is readily seen that it is SC-continuous because $\phi$, given by (24), is smooth; $F$ is $C$-continuous a.e. since $\phi^{\prime}=F$ a.e.; and $\operatorname{SCD} F=F$ since $D^{2} \phi=f$.

It is easy to extend Definition 7(c) to define upper and lower SC-derivatives of $F$ at $x, u \operatorname{SC} D F(x)$, and $l \mathrm{SC} D F(x)$ respectively. Then following the discussion in Paragraph 8 this new derivative can be used to define a new Perron integral provided the two conditions (i) and (ii) of Paragraph 8, are satisfied. That this is so follows from classical properties of convex functions since, in short, to say $F$ is monotonic is equivalent to saying $\phi$ is convex; see Burkill [7]. Since the development follows that of Paragraph 4 it is sufficient to define the major functions.

Definition 8. Let $B \subset[a, b],|B|=b-a, a, b \in B, f: B \rightarrow \mathbb{R}$. Then $M$ is an SC-major function of $f$ with respect to the basis $B$ if $M=[a, b] \rightarrow \mathbb{R}$, $M \in \mathbf{P}(a, b)$ and

(a) $M$ is SC-continuous,

(a) $2 M$ is $C$-continuous at all points of $B$,

(b) $M(a)=0$,

(c) $l \mathrm{SC} D M \geqslant f$ a.e.,

(d) lSC $D M>-\infty$ n.e. 
If then $f \in \mathbf{S C P}(a, b)$ with basis $B$ and $x \in B$ then $f \in \operatorname{SCP}(a, x)$ with basis $B \cap[a, x]$, and if $F(x)=\operatorname{SCP} \int_{a}^{x} F$ then $\operatorname{SCD} F=F$ a.e. If $f \in \mathrm{SCP}$ with basis $B_{1}$, and with basis $B_{2}$, then $f \in \mathbf{S C P}$ with basis $B_{1} \cap B_{2}$, and the primitives agree on $B_{1} \cap B_{2}$; so reference to $B$ will, when convenient, be omitted. Further, from the above discussion, and that in paragraph 2 , if $f$ is given by (2) and $a$ is a point of convergence of (5), then $f \in \operatorname{SCP}(a, a+2 \pi)$ with basis the set of points of convergence of (5). Further, we get (4);

$$
\operatorname{SCP} \int_{a}^{a+\pi} f=F(a+2 \pi)-F(a)=\pi a_{0}
$$

and, as in Paragraph 2, if we had an integration by parts formula we could obtain (7).

It is clear that the concept of $C$-continuity can be extended to define $C$-differentiability, the $C$-derivative of $F$ and $x$ being $C D F(x)$, with $\mathrm{uCDF}(x)$, $\operatorname{ICDF}(x)$ having the obvious meanings; then along the lines of Definition 1 we can define a CP-integral; see Burkill [6].

14. The $\mathrm{P}^{2}$-integral. A simpler integral to define than the SCP-integral is suggested by the above discussion, using $D^{2} \phi$ rather than $\mathrm{SCDF}$ as the starting point. Although a second order integral is then obtained the development follows that of Definition 1 except that now the requirement that $M-m$ be monotonic, Paragraph 8 (ii), is replaced by $M-m$ being convex. In this way we obtain the James $\mathrm{P}^{2}$-integral; see Gage and James [12], James [18]. As in Paragraph 13 we only define the major functions.

Definition 9. Let $f:[a, b] \rightarrow \mathbb{R}$. Then $M$ is a $\mathbf{P}^{2}$-major if $M:[a, b] \rightarrow \mathbb{R}$ and

(a) $M$ is continuous and smooth,

(b) $M(a)=M(b)=0$,

(c) $\mathbf{I D}^{2} M \geqslant f$ a.e.,

(d) $I D^{2} M>-\infty$ n.e.

As might be expectedd, if $\phi=\mathrm{P}^{2} \int f$ then $D^{2} \phi=f$ a.e.; in addition $\phi$ is continuous, smooth, ACG, and $\phi^{\prime}$ exists a.e. and is $D$-integrable; see James [18], Sklyarenko [35]. Further the above discussion suggests a close connection between the $\mathrm{P}^{2}$ - and SCP-integrals; this is given by the following theorem due to Cross [8], and Sklyarenko [36].

Theorem 10. $f \in \operatorname{SCP}(a, b)$ with basis $B$ if and only if $f \in \mathbf{P}^{2}(a, b)$ and

(i) if $\phi=\mathbf{P}^{2} \int f$ then $\phi$ is $\mathrm{ACG}^{*}$;

(ii) $\phi_{+}^{\prime}(a), \phi_{-}^{\prime}(b)$ exist.

If $F=\operatorname{SCP} \int f$, then

$$
\phi(x)=\phi(a)+\mathrm{P} \int_{a}^{x} F-\frac{x-a}{b-a} \mathrm{P} \int_{a}^{x} F .
$$


15. Integration by parts for the SCP-integral. The following result was stated by Burkill, [7].

THEOREM 11. Let $f \in \operatorname{SCP}(a, b)$ with basis $B, F=\operatorname{SCP} \int f$, and $g$ continuous and of bounded variation, $G=\int g$. Then $f G \in \operatorname{SCP}(a, b)$ with basis $B$, and for all $\alpha, \beta \in B$

$$
\operatorname{SCP} \int_{\alpha}^{\beta} f G=F(\beta) G(\beta)-F(\alpha) G(\alpha)-\mathrm{P} \int_{\alpha}^{\beta} F g .
$$

No proof was given but it was suggested that one could be given along the lines of the proof of the analogous result for the CP-integral (Burkill [6]). (The result for the CP-integral is, except for obvious modifications the same as Theorem 11 but $g$ is not required to be continuous.)

The conditions of Theorem 11 imply the existence of the right-hand side of (26); to see this let $M$ be any SCP-major function $f$; then since $F=M+(F-$ $M)$ and $M \in \mathbf{P}, F-M$ increasing and bounded and so P-integrable, it follows that $F \in \mathbf{P}(a, b)$; hence, by Theorem 4, $F g \in \mathbf{P}(a, b)$. Hence, arguing as for (9), it is sufficient to prove that $f G+F g \in \mathbf{S C P}$ and

$$
\operatorname{SCP} \int_{\alpha}^{\beta}(f G+F g)=F(\beta) G(\beta)-F(\alpha) G(\alpha) ;
$$

and for this an analogue of (13) is needed for SC-differentiation. In the case of $C$-derivation Burkill had obtained such an analogue and used it to prove the analogue of (27) (Burkill [6]).

LEMMA 12. If $G\left(x_{0}\right)>0$ and $G^{\prime}$ is bounded near $x_{0}$ then

$$
\operatorname{ICD}(M G)\left(x_{0}\right)=M\left(x_{0}\right) G^{\prime}\left(x_{0}\right)+G\left(x_{0}\right) \operatorname{ICD} M\left(x_{0}\right) .
$$

It was noted by $\mathrm{C}$. M. Lee that Lemma 12 does not extend to SC-differentiation; he gave the following example. Take

$$
M(x)= \begin{cases}=|x|^{-1 / 2}, & x \neq 0 ; G(x)=x \\ =0, & x=0 .\end{cases}
$$

Then the left-hand side of $(28)$ is $-\infty$, while the right-hand side is 0 . This example shows that for SC-differentiation even a weaker form of (28) is false, namely that, under the conditions of Lemma 12

$$
\operatorname{ISCD}(M G)\left(x_{0}\right) \geqslant M\left(x_{0}\right) G^{\prime}\left(x_{0}\right)+G\left(x_{0}\right) \operatorname{ISCDM}\left(x_{0}\right) ;
$$

this would be sufficient to prove that if $M$ is a SCP-major function of $f$ then $M G$ is an SCP-major function of $f G+F g$. 
This error had been noted earlier by a Soviet mathematician, who pointed it out to Burkill [7, corrigendum]. In 1974 Bullen and Mudhopadhyay [5] obtained a correct version of Lemma 12 for the SC-derivative.

LeMma 13. Let $M$ be defined a.e. in $[a, b], M \in \mathbf{P}(a, b)$ and SC-continuous at $x_{0}$, and such that

$$
\begin{aligned}
-\infty & <\mathbb{I} M\left(x_{0}\right)=\liminf _{h \rightarrow 0} \frac{1}{2 h} \mathbf{P} \int_{x_{0}}^{x_{0}+h} M \leqslant \mathbf{u} M\left(x_{0}\right) \\
& =\limsup _{h \rightarrow 0} \frac{1}{2 h} \mathbf{P} \int_{x_{0}-h}^{x_{0} h} M<\infty ;
\end{aligned}
$$

let $G$ have a continuous derivative $g$ in some neighbourhood of $x_{0}, G\left(x_{0}\right) \geqslant 0$, $g\left(x_{0}\right) \geqslant 0$. Then

$$
\operatorname{ISCD}(M G)\left(x_{0}\right) \geqslant\left(2 \mathbf{I} M\left(x_{0}\right)-\mathbf{u} M\left(x_{0}\right)\right) g\left(x_{0}\right)+G\left(x_{0}\right) \operatorname{ISCDM}\left(x_{0}\right) .
$$

Using Lemma 13 they were able to prove Theorem 11 under the additional hypothesis

$$
-\infty<\mathbf{l} F \leqslant \mathbf{u} F<\infty \text { n.e. }
$$

Unfortunately for the application to trigonometric series, if $F$ is given by (5) it is not known whether (29) holds; equivalently, it is not known if

$$
-\infty<1 D^{2} \phi \leqslant \mathbf{u} D^{2} \phi<\infty \quad \text { n.e., }
$$

where $\phi$ is given by (24).

In 1981 Sklyarenko [36] gave a proof of Theorem 11. He first showed that the hypotheses of Theorem 11 give that $f G \in \mathbf{P}^{2}(a, b)$ with $\mathbf{P}^{2}$-primitive

$$
\begin{aligned}
R(x)= & \int_{a}^{x} F G-\int_{a}^{x} \int_{a}^{t} f(u) g(u) d u d t \\
& -\frac{x-a}{b-a}\left\{\int_{a}^{b} F G-\int_{a}^{b} \int_{a}^{t} F(u) g(u) d u d t\right\}
\end{aligned}
$$

(where all the integrals are P-integrals); further for all $x \in B, R^{\prime}(x)$ exists. Hence, by Theorem 10, $f G \in \mathbf{S C P}$ and using (25), (29) leads to (26).

\section{References}

[1] A. Aleksandrov, 'Über die Äquivalenz des Perronschen und des Denjoyschen Integralbegriffes', Math. Z. 20 (1924), 213-222.

[2] H. Bauer, 'Der Perronschen Integralbegriff und seine Beziehung zum Lebesgueschen', Monatsch. Math. Phys. 26 (1915), 153-198. 
[3] Andrew M. Bruckner, Differentiation of real functions (Lecture Notes in Math. 659, SpringerVerlag, New York, 1978).

[4] P. S. Bullen 'Non-absolute integrals: a survey', Real Anal. Exchange 5 (1980), 195-259.

[5] P. S. Bullen and S. N. Mukhopadhyay, 'Integration by parts formula for some trigonometric integrals', Proc. London Math. Soc. (3) 28 (1974), 159-173.

[6] J. C. Burkill, 'The Cesàro-Perron scale of integration', Proc. London Math. Soc. (2) 39 (1935), 541-552.

[7] J. C. Burkill, 'Integrals and trigonometric series', Proc. London Math. Soc. (3) 1 (1951), 46-57. Corrigendum, Proc. London Math. Soc. (3) 46 (1983), 190.

[8] G. Cross, 'A relation between two symmetric integrals', Proc. Amer. Math. Soc. 14 (1963), $185-190$.

[9] Arnaud Denjoy, 'Une extension de l'intégrale de M. Lebesgue', C. R. Acad. Sci. Paris 154 (1912), 859-862.

[10] Arnaud Denjoy, Leçons sur le calcul de coefficients d' une série trigonomètrique I-IV (GauthierVillars, Paris, 1941-1949).

[11] Arnaud Denjoy, Mémoire sur la dérivation et son calcul inverse (Gauthier-Villars, Paris, 1954).

[12] W. H. Gage and R. D. James, 'A generalized integral', Trans. Roy. Soc. Canada III(3) 40 (1946), 25-35.

[13] L. Gordon and S. Lasher, 'An elementary proof of integration by parts for the Perron integral', Proc. Amer. Math. Soc. 18 (1967), 394-398.

[14] H. Hake, 'Über de la Vallée Poussins Ober-und Unterfunktionen', Math. Ann. 83 (1921), 119-142.

[15] R. Henstock, 'Integration by parts', Aequationes Math. 9 (1973), 1-18.

[16] E. W. Hobson, The theory of functions of a real variable (Cambridge University Press, 3rd ed., 1927).

[17] C. T. Ionescu-Tulcea, 'Sur l'intégration des nombres derivés', C. R. Acad. Sci. Paris 225 (1949), 558-560.

[18] R. D. James, 'A generalized integral II', Canad. J. Math. 2 (1950), 297-306.

[19] R. D. James, 'Integrals and summable trigonometric series', Bull. Amer. Math. Soc. 61 (1955), $1-15$.

[20] R. L. Jeffrey, 'Perron integrals', Bull. Amer. Math. Soc. 48 (1942), 714-1-717.

[21] H. Lebesgue, Leçons sur l'intégration et la recherche des fonctions primitives (2nd ed., Paris, 1926).

[22] H. Looman, 'Über die Perronsche integral definition', Math. Ann. 93 (1935), 153-156.

[23] N. N. Luzin, 'Sur les propriétés de l'intégrale de M. Denjoy', C. R. Acad. Sci. Paris 155 (1912), 1475-1478.

[24] J. Mařik, 'Základy theorie integrálu v euklidových prostorech, I-III', Časopis Pěst. Mat. 77 (1952), 1-51, 125-145, 267-230.

[25] Edward James McShane, 'On Perron integration', Bull. Amer. Math. Soc. 48 (1942), 718-726.

[26] Edward James McShane, Integration (Princeton University Press, 1944).

[27] I. P. Natanson, Theory of functions of a real variable (2nd ed., revised, New York, 1960).

[28] O. Perron, 'Über den Integralbelgriff, Sitzber', Heidelberg Akad. Wiss. Abt. A 16 (1914), 1-16.

[29] I. N. Pesin, Classical and modern integration theory (New York, 1970).

[30] W. Pfeffer, 'Integration by parts for the generalized Riemann-Stieltjes integral', J. Austral. Math. Soc. ( Series A) 34 (1983), 229-233.

[31] J. Ridder, 'Ueber Definitionen von Perron-Integralen', I, II, Indag.Math. 9 (1947), 227-235, 280-289; MR 8, 506; 9, 19.

[32] S. Saks, Theory of the integral, (2nd ed., revised, New York, 1937) Zbl. 17, 300.

[33] W. L. C. Sargent, 'On the integrability of a product', J. London Math. Soc. 23 (1948), 28-34. MR 10, 108.

[34] D. N. Sarkhel, 'A criterion for Perron integrability', Proc. Amer. Math. Soc. 7 (1978), 109-112. MR 58 \#17006. 
[35] V. A. Sklyarenko, 'Some properties of the $P^{2}$-integral', Mat. Zametki 12 (1972), 693-700; English transl., Math. Notes 12 (1974), 856-860 (1973). MR 47 \#8785.

[36] V. A. Sklyarenko, 'On integration by parts for Burkill's SCP-integrals', Mat. Sb. 112 (1980), 630-646; English transl., Math. USSR Sbornik 40 (1981), 567-583. MR 81k, 26009.

[37] G. P. Tolstov, 'Sur l'intégrale de Perron', Mat. Sb. 5 (1939), 647-659: 1. 208.

[38] A. Zygmund, Trigonometrical series, (Cambridge Univ. Press, 1959). MR 21 \#6498.

Department of Mathematics

The University of British Columbia

Vancouver B. C. V6T 1W5

Canada 